\title{
Correction to: Identification of novel mutations in congenital afibrinogenemia patients and molecular modeling of missense mutations in Pakistani population
}

\author{
Arshi Naz ${ }^{1 *}$ D, Arijit Biswas², Tehmina Nafees Khan ${ }^{1}$, Anne Goodeve $^{3}$, Nisar Ahmed ${ }^{4}$, Nazish Saqlain ${ }^{4}$, \\ Shariq Ahmed ${ }^{1}$, Ikram Din Ujjan ${ }^{5}$, Tahir S. Shamsi ${ }^{1}$ and Johannes Oldenburg ${ }^{2}$
}

\author{
Correction to: Thromb J (2017) 15:24 \\ https://doi.org/10.1186/s12959-017-0143-3
}

Following the publication of this article [1], the authors noted the following typographical errors:

1) Affiliation 3 should read "University of Sheffield, Sheffield, United Kingdom" and Affiliations 6, 7, 8 and 9 were unnecessary duplicates

2) In the abstract the sentence "Ten patients had mutations in FGA followed by three mutations in FGB and three mutations in FGG, respectively" should be "Ten patients had mutations in FGA followed by four mutations in $F G B$ and two mutations in $F G G$, respectively."

3) In the Results section the following three sentences:

"In FGA gene, eight mutations were identified as novel and the remaining two were reported mutations. Eight novel mutations include five missense, one nonsense and two frameshift mutations including homozygous and a compound heterozygous frameshift mutation. The two nonsense mutations in FGA are reported in literature. There is one more mutation with reported status in proband (C3). This patient had compound heterozygous mutation with frameshift as novel mutation and nonsense as reported. We identified three mutations in FGB including one novel missense mutation (C9) and two homozygous nonsense mutations reported in siblings. The FGG gene mutations are the rarest of all three fibrinogen genes. We detected three novel mutations

\footnotetext{
* Correspondence: labarshi@yahoo.com; labarshi2013@gmail.com ${ }^{1}$ National Institute of Blood Diseases and Bone Marrow Transplantation, Karachi, Pakistan

Full list of author information is available at the end of the article
}

including two similar nonsense mutations in siblings and one frameshift mutation in unrelated proband in different exons of FGG gene (Table 1)."

Should be:

"In FGA gene, seven mutations were identified as novel and the remaining three were reported mutations. Seven novel mutations include five missense and two frameshift mutations including homozygous and a compound heterozygous frameshift mutation. The three nonsense mutations in FGA are reported in literature. There is one more mutation with reported status in proband (C3). This patient had compound heterozygous mutation with frameshift as novel mutation and nonsense as reported. We identified four mutations in FGB including one novel missense mutation (C9), two homozygous nonsense mutations reported in siblings and one frameshift mutation(C12). The FGG gene mutations are the rarest of all three fibrinogen genes. We detected two novel similar nonsense mutations in siblings (Table 1)."

4) There are a number of errors in Tables 1 and 2. The corrected versions are provided in this Correction article with the corrections given in bold.

5) Frameshift mutation (p.Gln282Thr fsx83*) and (p. Lys (AAA) 48Arg fs9*) are the novel compound heterozygous mutations which have manifested deletions along with frameshift defects" should in fact read "Frameshift mutations (p.Thr283Arg fs $138^{*}$ ) and (p. Lys (AAA) 48Arg fs9*) are the novel compound heterozygous mutations which have manifested deletions along with frameshift defects. 
Table 1 Genotypic expression of mutations in fibrinogen gene (FGA, FGB \& FGG)

\begin{tabular}{|c|c|c|c|c|c|c|c|}
\hline IP \# & Gene & Exon & Mutation & Amino Acid change & Zygosity & Mutation type & Reported/Novel \\
\hline C1 & $F G A$ & 1 & c. $24 \mathrm{C}>\mathrm{A}$ & p.Cys8* & Homozygous & Nonsense & Ref \#23 $\epsilon^{€}$ \\
\hline C2 & & 2 & c.143_144 del AA & p.Lys (AAA)48Arg fs9* & Compound Heterozygous & Frame shift & Novel mutation \\
\hline \multirow[t]{2}{*}{ C3 } & & 5 & c.846delG & p.Thr 283Arg fs $138^{*}$ & Compound Heterozygous & Frame shift & Novel mutation \\
\hline & & 4 & c. $385 C>T$ & p.Arg129* & Homozygous & Nonsense & Ref $\# 24^{\epsilon}$ \\
\hline $\mathrm{C} 4$ & & 4 & c.385 C > T & p.Arg129* & Homozygous & Nonsense & Ref \#24 $€$ \\
\hline C5 & & 5 & C. $598 \mathrm{C}>\mathrm{T}$ & p.Gln $200^{*}$ & Homozygous & Nonsense & $\operatorname{Ref} 27^{*}$ \\
\hline C6 & & 5 & c. $.904 C>G$ & p.Pro302Ala & Homozygous & Missense & Novel mutation \\
\hline C7 & & 5 & c. $913 A>G$ & p.Thr 305 Ala & Homozygous & Missense & Novel mutation \\
\hline $\mathrm{C} 8$ & & 5 & c. $.992 A>G$ & p.Thr331Ala & Homozygous & Missense & Novel mutation \\
\hline$C 9^{\mathbf{i}}$ & & 5 & c. $992 A>G$ & p.Thr331Ala & Homozygous & Missense & Novel mutation \\
\hline C10 & & 5 & c. $.973 A>G$ & p.Ser325Gly & Homozygous & Missense & Novel mutation \\
\hline C11A & $F G B$ & 2 & c. $141>\mathrm{T}$ & p.Arg47* & Homozygous & Nonsense & Ref \# $25^{\epsilon}$ \\
\hline $\mathrm{C} 11 \mathrm{~B}$ & & 2 & c. $141 \mathrm{C}>\mathrm{T}$ & p.Arg47* & Homozygous & Nonsense & Ref \# $25^{\epsilon}$ \\
\hline$C 9^{i i}$ & & 8 & c. $1294 \mathrm{~T}>\mathrm{A}$ & p.Trp 432Arg & Homozygous & Missense & Novel mutation \\
\hline $\mathrm{C} 12$ & & 2 & c.118_124dupTTCTTCA & TTCTTCA & Homozygous & Frame shift & Novel mutation \\
\hline C13A & FGG & 4 & c.361A > T & p.Lys $121^{*}$ & Homozygous & Nonsense & Novel mutation \\
\hline C13B & & 4 & c. $361 \mathrm{~A}>\mathrm{T}$ & Lys121* & Homozygous & Nonsense & Novel mutation \\
\hline
\end{tabular}

Identified novel and reported mutations in three genes of fibrinogen. The letter A and B with patient code designate the sibling status, $\mathbf{i} \&$ ii shows mutation identified in same patient but in different genes, $€$ (repor ted mutation) c (complimentary deoxyribonucleic acid), A (adenine), $T$ (thymine), $C$ (cytosine), $G$ (guanine), Lys (lysine), Arg (arginine), Tyr (tyrosine), Pro (proline), Trp (tryptophan), Thr (threonine), Gln (glycine), Cys = cystine, fs = frame shift, * stop codon number, FGA (fibrinogen Aa-chain gene), FGB (fibrinogen Bß-chain gene), FGG (fibrinogen GY-chain gene.

Table 2 Assessment of coagulation markers and bleeding scores with consanguinity/ethnicity

\begin{tabular}{|c|c|c|c|c|c|c|c|c|}
\hline IP\# & $\begin{array}{l}\text { Fibrinogen } \\
\text { Level } \\
(\mathrm{g} / \mathrm{l})\end{array}$ & $\begin{array}{l}\text { Thrombin } \\
\text { Time } \\
\text { (Sec) }\end{array}$ & $\begin{array}{l}\text { Prothrombin } \\
\text { Time } \\
\text { (Sec) }\end{array}$ & $\begin{array}{l}\text { Activated partial thromboplastin Time } \\
\text { (aPTT) (Sec) }\end{array}$ & $\begin{array}{l}\text { Bleeding } \\
\text { Score }\end{array}$ & Consanguinity & $\begin{array}{l}\text { Interfamilial } \\
\text { Relation }\end{array}$ & $\begin{array}{l}\text { Ethnic } \\
\text { Origin }\end{array}$ \\
\hline${ }^{*} \mathrm{C} 1$ & 0.01 & 23 & $>120$ & $>180$ & 20 & positive & Unrelated & NA \\
\hline C2 & 0.02 & 24 & $>120$ & $>180$ & 21 & positive & Unrelated & Punjabi \\
\hline C3 & 0 & 33 & $>120$ & $>180$ & 22 & positive & Unrelated & Punjabi \\
\hline C4 & 0.1 & 24 & $>120$ & $>180$ & 17 & positive & Unrelated & $\begin{array}{l}\text { Urdu } \\
\text { Speaking }\end{array}$ \\
\hline C5 & 0.02 & 31 & $>120$ & $>180$ & 20 & positive & Unrelated & Sindhi \\
\hline C6 & 0.01 & 25 & $>120$ & $>180$ & 20 & positive & Unrelated & $\begin{array}{l}\text { Urdu } \\
\text { speaking }\end{array}$ \\
\hline C7 & 0.02 & 29 & $>120$ & $>180$ & 22 & positive & Unrelated & Sindhi \\
\hline C8 & 0.0 & 30 & $>120$ & $>180$ & 20 & positive & Unrelated & Sindhi \\
\hline C9 & 0.0 & 32 & $>120$ & $>180$ & 22 & positive & Unrelated & Punjabi \\
\hline $\mathrm{C} 10$ & 0.01 & 25 & $>120$ & $>180$ & 16 & positive & Unrelated & Punjabi \\
\hline C11A & 0.02 & 28 & $>120$ & $>180$ & 18 & positive & $* *$ & Punjabi \\
\hline $\mathrm{C} 11 \mathrm{~B}$ & 0.01 & 24 & $>120$ & $>180$ & 16 & positive & & Punjabi \\
\hline $\mathrm{C} 12$ & 0.0 & 30 & $>120$ & $>180$ & 21 & positive & Unrelated & Punjabi \\
\hline C13A & 0.02 & 24 & $>120$ & $>180$ & 20 & positive & $* *$ & Punjabi \\
\hline C13B & 0.01 & 25 & $>120$ & $>180$ & 21 & positive & & Punjabi \\
\hline
\end{tabular}

Shows the individual test values of PT, aPTT and fibrinogen (Clauss Method), consanguinity and the relationship status. Bleeding score calculated, Tosetto et al [26]. ** Siblings, NA = not available, s (seconds). The fibrinogen levels in all patients were found to be equal to or lower than $0.1 \mathrm{~g} / \mathrm{l}$ (Normal Range $2-4 \mathrm{~g} / \mathrm{dl}$ ), PT more than $120 \mathrm{~s}$ (Normal Range 9-11 s) aPTT more than $180 \mathrm{~s}$ (Normal Range 24-27 s) and prolonged thrombin time (normal range 10-13 s). Ethnicity explains the frequency of majorly affected, thickly populated and largest province of Pakistan (Punjab). 


\section{Author details}

${ }^{1}$ National Institute of Blood Diseases and Bone Marrow Transplantation,

Karachi, Pakistan. ${ }^{2}$ Institute of Experimental Hematology and Transfusion Medicine, Bonn, Germany. ${ }^{3}$ University of Sheffield, Sheffield, UK. ${ }^{4}$ Children's Hospital, Lahore, Pakistan. '5iaquat university of medical and health sciences, Jamshoro, Pakistan.

Received: 6 March 2019 Accepted: 6 March 2019

Published online: 04 April 2019

\section{Reference}

1. Naz A, et al. Identification of novel mutations in congenital afibrinogenemia patients and molecular modeling of missense mutations in Pakistani population. Thromb J. 2017;15:24. https://doi.org/10.1186/s12959-017-0143-3. 